Document downloaded from:

http://hdl.handle.net/10251/49691

This paper must be cited as:

Salvador Rubio, FJ.; Gimeno García, J.; Pastor Enguídanos, JM.; Martí Aldaraví, P. (2014). Effect of turbulence model and inlet boundary condition on the Diesel spray behavior simulated by an Eulerian Spray Atomization (ESA) model. International Journal of Multiphase Flow. 65:105-116. doi:10.1016/j.ijmultiphaseflow.2014.06.003.

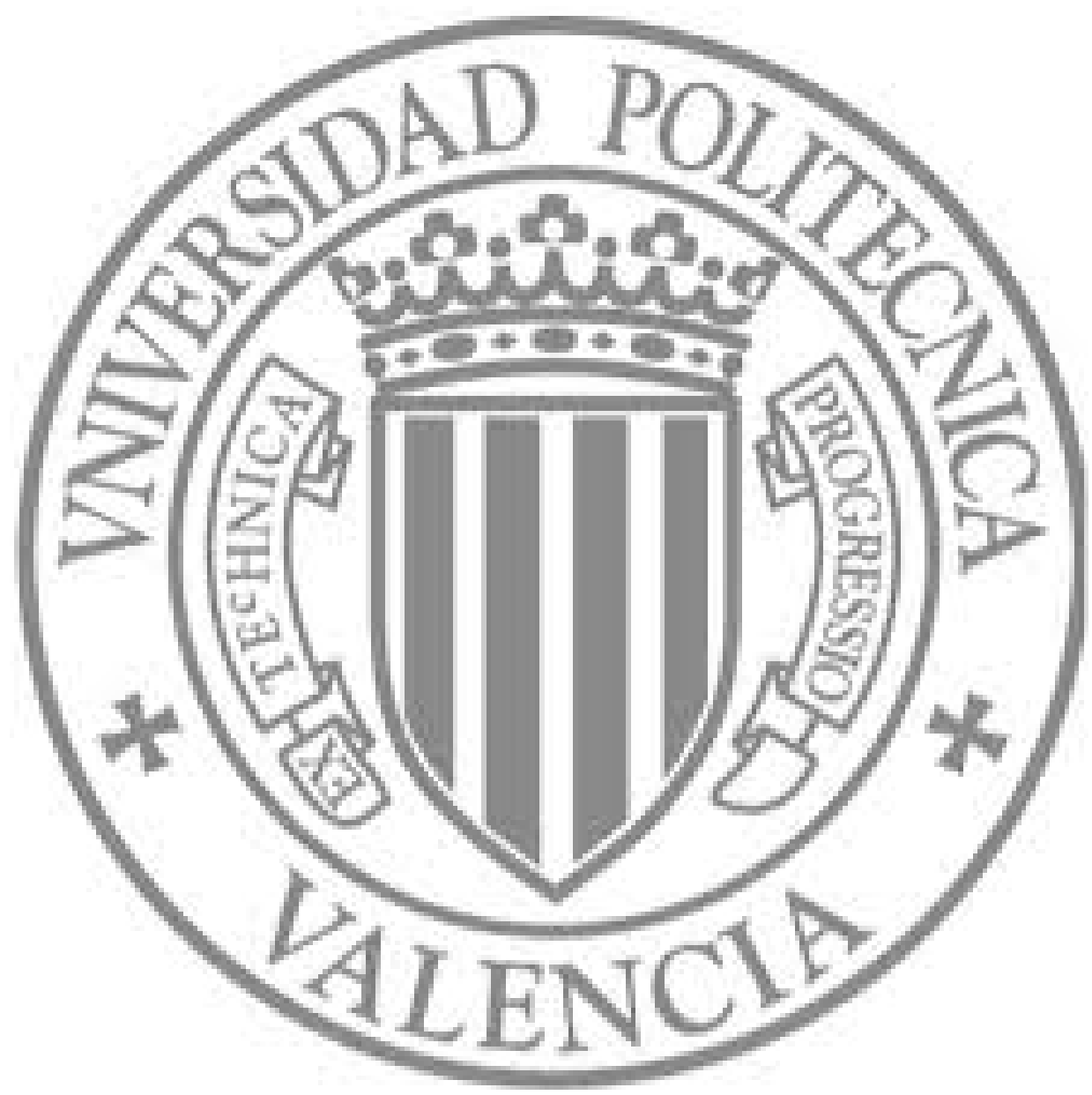

The final publication is available at

http://dx.doi.org/10.1016/j.ijmultiphaseflow.2014.06.003

Copyright Elsevier 


\title{
Effect of turbulence model and inlet boundary condition on the Diesel spray behavior simulated by an Eulerian Spray Atomization (ESA) model
}

\author{
Francisco Javier Salvador, Jaime Gimeno, José Manuel Pastor, Pedro \\ Martí-Aldaravi* \\ CMT - Motores Térmicos, Universitat Politècnica de València, Edificio 6D, Camino de \\ Vera s/n, 46022, Valencia, Spain. Tel. num. 0034-963877650. Fax Num. \\ 0034-963877659
}

\begin{abstract}
Simulating liquid spray first and second atomization is not an easy task. Many models have been developed over the past years, but Eulerian ones have proved their better performance for the dense zone of the spray. In this work a new compressible Eulerian model is used to compute the internal flow together with the spray. Up to five two-equation turbulence models have been tested and its influence is remarkable in terms of spray behavior, but also greatly affects the mass flow rate and the momentum flux. At the end, SST $k-\omega$ model proves to be best than the others. Additionally, different types of inlet boundary conditions have been also tested and analyzed. Results when compared with previously obtained experimental data show that the commonly used for external flow time-varying velocity boundary condition gives also good performance for the internal flow.
\end{abstract}

Keywords: Air-fuel mixing, OpenFOAM, Eulerian, inlet, turbulence, fuel injection

\footnotetext{
${ }^{*}$ Corresponding author

Email addresses: fsalvado@mot.upv.es (Francisco Javier Salvador), jaigigar@mot.upv.es (Jaime Gimeno), jopasen@mot.upv.es (José Manuel Pastor), pedmar15@mot.upv.es (Pedro Martí-Aldaraví)
} 


\section{Introduction}

Understanding and being able to predict fuel break-up during the injection process is a key in order to improve air-fuel mixing and hence reduce the fuel consumption of an internal combustion engine. With this objective, many experimental and computational studies have been carried out for decades. Among all fuel injection applications in the industry, this work focuses on common-rail diesel injectors, then high injection pressures, high velocities and small droplets are the main characteristics of the liquid spray.

Liquid atomization or break-up is usually modeled through a Discrete Droplet Model (DDM) under a Lagrangian framework. This model, although commonly used nowadays (e.g. Battistoni et al. (2012); Lee and Reitz (2013)), only works fine in absence of liquid ligaments and if liquid droplets are dispersed. However Vallet et al. (2001) developed an Eulerian model, called from now $\Sigma-Y$ model, able to use the advantages of an Eulerian framework for the dense part. Other authors, Blokkeel et al. (2003) and Lebas et al. (2005), modified that model by adding a switch to Lagrangian framework when liquid droplets are dispersed enough; this model was called Eulerian-Lagrangian Spray Atomization (ELSA). These models and their further improvements have proved its reliability in simulating diesel fuel injection processes under different conditions in works presented by Ning et al. (2009); Lebas et al. (2009); Hoyas et al. (2013) and García-Oliver et al. (2013).

On the other hand, it is well known that the nozzle geometric parameters have a great influence on the spray behavior, as experimentally proved by Desantes et al. (2005) and Bermúdez et al. (2005) and computationally reproduced by Salvador et al. (2013). Thus, coupling internal and external flow simulations leads to a better representation of reality. This is usually done by a two-step methodology, i.e. Battistoni et al. (2012); Som et al. (2010). In such methodologies, internal flow spatial and temporal distributions of all fields (velocity, turbulent kinetic energy, dissipation rate, void fraction...) are transferred (and some times averaged) from the internal flow simulation to a primary break-up (blob) model which uses them to initialize droplet properties within the hole area of the Lagrangian external flow simulation.

This coupling methodology has several issues to be solved. For example, a sort of mapping procedure has to be implemented to spatially distribute blobs and its physical quantities of the primary break-up model inside the nozzle area. Also, computational time steps are much different (on the order or $10^{-8} \mathrm{~s}$ for nozzle flow and $10^{-6} \mathrm{~s}$ for spray as commented by Battistoni 
et al. (2012)), thus a time interpolation is required.

There are other options to model the multi-phase internal and external flows of fuel injection applications. Marcer et al. (2000) used a VolumeOf-Fluid (VOF) interface tracking approach to simulate the cavitation phenomenon inside the nozzle and also the flow downstream the injector exit. Recently, Arienti and Sussman (2014) validated a combined level-set volumeof-fluid model to calculate internal and external flows seamlessly across an injection orifice. However, as they conclude, a very high resolution grid is needed to provide a realistic turbulent flow at high Reynolds numbers and the stable time-step is several orders of magnitude smaller than a full injection period, issues which increase a lot the computational cost.

The best solution to overcome issues of coupling and still being able to simulate the effects of nozzle parameters (rounding radius at the orifice entry, exit diameter...) on the spray behavior is simple: using the $\Sigma-Y$ model of Vallet et al. (2001) to simulate internal and external flows in one unique domain. Notwithstanding, this methodology also has some limitations or drawbacks: computational time steps and mesh resolutions characteristic of nozzle flows are small, so the computational cost increases quite a lot; ambient conditions are limited to the range where hypothesis of $\Sigma-Y$ model are true; and no cavitation nor evaporation (process studied by García-Oliver et al. (2013) for the $\Sigma-Y$ model) models are included so far because a third phase, fuel vapor, would be necessary.

As this is the first time that diesel injection internal and external flows are going to be simulated at the same time, and both flows are of quite different nature, a review of most suitable boundary conditions and turbulence models is necessary. And that is the main objective of this paper. Three types of inlet boundary conditions are tested: constant pressure, non-reflective pressure, and time-varying velocity. For one of those inlet boundaries, concretely non-reflective pressure, five different RANS turbulence models are tested: standard $k-\epsilon$, high-density ratio $k-\epsilon$ (Demoulin et al. (2007)), RNG $k-\epsilon$, Realizable $k-\epsilon$ and SST $k-\omega$.

In order to validate the new solver and also to select the best turbulence model and inlet condition, comparison with experimental data is necessary. A single-hole conical nozzle geometry with an exit diameter of $112 \mu \mathrm{m}$ is selected to avoid the uncertainties related to cavitation and spray-spray interaction as done by Desantes et al. (2005) and Payri et al. (2005). Available data for such nozzle are: mass flow rate measurements, momentum flux experimental measurements and diffusive back-light spray visualization un- 
der non-cavitating and non-evaporative conditions. These measurements are presented and discussed in the work of Payri et al. (2011b, 2012). Only one experimental point of the whole test matrix corresponding to low injection pressure and high ambient density is selected to keep the number of simulations within a reasonable limit.

\section{Methodology}

\subsection{Code description}

An Eulerian homogeneous unsteady multi-phase model is developed and used to simulate the atomization. This new solver is the same than the one developed by Vallet et al. (2001) except for three important matters: the common pressure-equation (see Weller et al. (1998)) is used instead of the equation of state of Vallet et al. (2001) or an isentropic relationship like the one used by Ning et al. (2009); the new model is compressible so the energy equation is required; and a PIMPLE algorithm (Corp. (2012)) is preferred rather than the common PISO algorithm.

Although the model is described in detail by Vallet et al. (2001); Demoulin et al. (2007); García-Oliver et al. (2013) and Beheshti et al. (2007), the basics are summarized in this paper. The key point of the original model is the striking analogy between atomization and turbulent mixing of a jet with a very large density difference with the ambient medium. Additionally, $\Sigma-Y$ model is based in four principles: (1) high Reynolds and Weber numbers, (2) the difference between the mean velocity of the liquid fluid and gaseous fluid particles can be calculated, (3) the dispersion of the liquid phase into the gas phase can be computed by a balance equation (Equation 1), and (4) the mean size of the liquid fragments can be calculated through the mean surface area of the liquid-gas interface per unit of volume (Equations 5 and 7).

The balance equation for the liquid mass fraction is Equation 1 (see also Vallet et al. (2001)). It can be seen that the break-up and mixing processes, due only to turbulence by hypothesis, are modeled with a diffusion flux closure term. Diffusivity coefficient $D_{Y, \text { eff }}$ is the one developed and tested by Demoulin et al. (2007) and adopted later by other authors such Belhadef et al. (2012). It is given in Equation 2 and is compounded by two terms. The first one follows the Fick's law, being $S c$ the Schmidt number, and corresponds to the diffusivity coefficient originally presented by Vallet et al. (2001). The second term represents the segregation that occurs between the heavy and 
the light phases because of the acceleration difference under a mean pressure gradient according to Demoulin et al. (2007). The modeling constant $C_{\rho}$ is equal to 1.8, value also set by Demoulin et al. (2007).

$$
\begin{aligned}
& \frac{\partial(\rho \cdot Y)}{\partial t}+\nabla \cdot(\Phi \cdot Y)-\nabla^{2}\left(D_{Y, \text { eff }} \cdot Y\right)=0 \\
& D_{Y, \text { eff }}=\frac{\mu_{t}}{S c}+C_{\rho} \frac{k^{2}}{\epsilon} \rho^{2}\left(\frac{1}{\rho_{g}}-\frac{1}{\rho_{l}}\right) Y(1-Y)
\end{aligned}
$$

Then, the density of the mixture can be calculated by the equation of state given in Equation 3 (Vallet et al. (2001)). The gas phase (Nitrogen for this study) is considered as perfect gas. The liquid, Standard Diesel Elite ${ }^{+}$, is compressible and its density is related to pressure and temperature by the relationship obtained by Payri et al. (2011a) and shown in Equation 4.

$$
\begin{gathered}
\rho=\frac{1}{\frac{Y}{\rho_{l}}+\frac{1-Y}{\rho_{g}}} \\
\rho_{l}=835.698-0.6280(T-298)+4.91410^{-7}\left(p-10^{5}\right)- \\
-7.049910^{-16}\left(p-10^{5}\right)^{2}+7.373910^{-4}(T-298)^{2}+ \\
+1.0363310^{-9}\left(p-10^{5}\right)(T-298)
\end{gathered}
$$

The balance equation for the mean inter-facial area per unit volume in the two-phase flow, developed by Vallet et al. (2001) and reviewed by Beheshti et al. (2007), is Equation 5. In this equation, $A$ is a production term related to the stretching of the gas-liquid interface by the mean velocity gradients, $a$ is the production term related to the stretching of the interface due to turbulence and droplet collision, and $V_{s}$ is the destruction term due to coalescence and ensures equilibrium (constant surface kinetic energy). As said by Vallet et al. (2001), these terms are included into the equation to take into account the physical phenomena responsible for stretching and collapse, and so the inter-facial area generation and destruction. The term $\Phi$, which also appears in Equation 1, is the mass flux through the cell faces. Concerning the diffusion coefficient $D_{\Sigma, \text { eff }}$, the simplest way is to use the classical Fick's law. It is given in Equation 6. This term is necessary because the interface is also dispersed by turbulence (Vallet et al. (2001)). Notice that diffusivity coefficients in Equations 1 and 5 are not exactly the same. Beheshti et al. 
(2007) also proceed the same way, they use the Fick's law for both coefficients with same turbulent diffusivity but different Schmidt numbers. This is due to the diffusion term in Equation 1 comes from an analogy of the closure term emerging from the averaging of the transport equation (Vallet et al. (2001); Demoulin et al. (2007)) meanwhile that term in Equation 5 is part of the transport equation.

$$
\begin{gathered}
\frac{\partial(\rho \Sigma)}{\partial t}+\nabla \cdot(\Phi \cdot \Sigma)-\nabla^{2}\left(D_{\Sigma, e f f} \cdot \Sigma\right)=(A+a) \rho \Sigma-V_{s} \rho \Sigma^{2} \\
D_{\Sigma, e f f}=\frac{\mu_{t}}{S c}
\end{gathered}
$$

If it is assumed that the flow is composed only of droplets with identical diameters, the Sauter Mean Diameter is easily computed from the values of liquid mass fraction, $Y$, and inter-facial surface, $\Sigma$, by Equation 7 obtained by Vallet et al. (2001).

$$
d_{32}=\frac{6 \rho Y}{\rho_{l} \Sigma}
$$

Though this quantity is computed in every simulation, no results of it are shown in the present paper and values of $A, a$ and $V_{s}$ terms are neither fully detailed because of several reasons: this is a preliminary study, this equation is not coupled with any other of the model, and a good study about this balance equation is the work of Beheshti et al. (2007). Furthermore, there is not experimental data of droplet size for the selected diesel injector and injection conditions, so even if results were shown, their reliability cannot be ensured.

The rest of balance equations (continuity, momentum, energy and pressure) are the common ones used in compressible solvers and can be easily found in the literature, e.g. Ph.D. Thesis of Weller et al. (1998).

All governing equations and two-fluid models are implemented and solved in the finite volume CFD code OpenFOAM 2.1.0 ${ }^{\circledR}$ Corp. (2012), which employs temporal and spatial discretization schemes that are bounded and preserve the proper physical limits on the fluid-dynamics variables. OpenFOAM library does not include any solver similar to the one described above, so a new one was created (by including all cited equations) and added to the library.

Note that there is not switch to a Lagrangian framework. This switch is controlled by the liquid mass fraction $Y$ or liquid volume fraction. Usually 
the transition is placed when $Y<0.01$, e.g. Hoyas et al. (2013), or when the volume fraction is lower than 50\%, e.g. Lebas et al. (2005), and only one parcel is generated per transition cell and per time step. Nevertheless, it will be seen that employing only an Eulerian approach also gives accurate results in terms of spray tip penetration, defined as the axial distance where $Y<0.001$. This definition was deeply discussed and established by the Engine Combustion Network (ECN) in a work carried out by Bardi et al. (2012). The definition of the spray angle is a topic still under discussion, however the criteria discussed and established by Desantes et al. (2005) is used, the slope of a linear fitting on the spray contour up to the $60 \%$ of the spray penetration.

As the Lagrangian description is not used, the current model is called from now on Eulerian Spray Atomization (ESA) model.

\subsection{Turbulence model}

There are many ways of modeling turbulence. In general, they can be classified in terms of degree of modelization (from highest to lowest): Reynolds-Averaged Navier-Stokes based (RANS or RAS), Large Eddy Simulation (LES), Detached Eddy Simulation (DES), and Direct Numerical Simulation (DNS). Discussions about which one is the most suitable in each case can be easily found in the literature. In this case, a RANS model is selected due to its simplicity (the present model is still under development) and grid independence (unlike LES and DNS approaches). Furthermore, for Eulerian-Eulerian multi-phase problems LES models have been only tested in VOF solvers, i.e. Befrui et al. (2012), and DNS is still under development, i.e. Duret et al. (2013). In addition, the computational cost is lower, so it is interesting from the industrial point of view.

RANS models can be also classified in three categories: linear eddy viscosity models, nonlinear eddy viscosity models, and Reynolds stress models. Again, discussions about which one is the most suitable in each case can be easily found in the literature. The employed transport model requires an eddy viscosity $\mu_{t}$ model. Also a linear constitutive relationship between the Reynolds stresses and the mean flow straining field is taken with the aim of "simplifying" the model. This linear relationship is also known as Boussinesq hypothesis.

At the same time, linear eddy viscosity models are classified according to the number of equations: algebraic equations or zero-equation models, 
where eddy viscosity is calculated directly from the flow variables; one equation models, which usually solve the turbulent kinetic energy; two equation models, which include two extra transport equations to represent the turbulent properties of the flow such turbulent kinetic energy $k$ and turbulent dissipation $\epsilon$ or specific dissipation $\omega$; etc. Two equation turbulence models are one of the most common type of turbulence models. Models like $k-\epsilon$ and $k-\omega$ models have become industry standard and are commonly used for most types of engineering problems. Although these models are still in active area of research, five of them they are selected for being tested in the current ESA model:

- Standard compressible $k-\epsilon$ model.

- High density ratio $k-\epsilon$ model (developed by Demoulin et al. (2007))

- Re-Normalization Group (RNG) $k-\epsilon$ model.

- Realizable $k-\epsilon$ model.

- Shear Stress Transport (SST) $k-\omega$ model.

These models are exactly the same than the ones used for mono-phase solvers. They model the turbulent kinetic energy and turbulent dissipation of the mixture, including the relative velocity as explained by Demoulin et al. (2007). The only model which has an additional term in the transport equations is the High density ration $k-\epsilon$ that adds a term into the $k$-equation to consider the shift of acceleration between the heavy fluid particles and the light fluid particles under the effect of the same pressure gradient.

\subsection{Computational domain and case setup}

The computational domain for the single-hole injector which is going to be simulated is shown in Figure 1. Due to symmetry of the geometry, the mesh covers only a sector of $5^{\circ}$ and is considered two-dimensional. Threedimensional simulations were performed and no significant differences were found in the results; however, if non-symmetric geometries are used (i.e. orifice offset), then the full three-dimensional domain is needed. Wedge bound-

aries are applied on the two sides planes, and symmetry boundary on the axis. The geometric details of the orifice and the test conditions are shown in Table 1. These test conditions give a Reynolds number of $R e=24845$, 
and Weber numbers of $W e_{l}=204400, W e_{g}=9570$, values large enough according to de la Morena (2011) to be in the range of validity of Vallet et al. (2001) model.

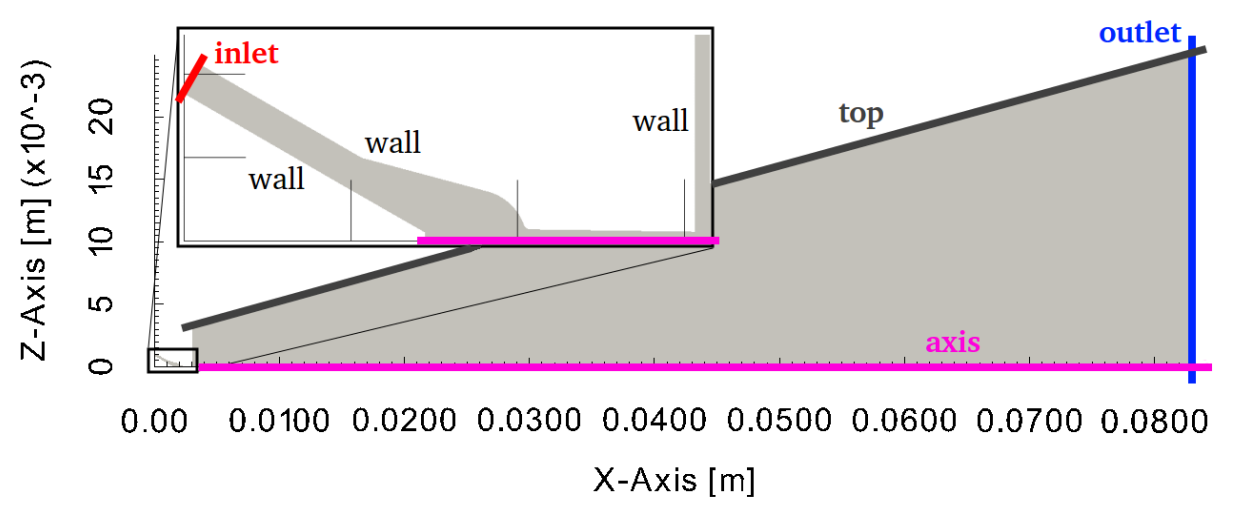

Figure 1: Computational domain for the simulations. Needle at maximum lift.

\begin{tabular}{lcc}
\hline Orifice inlet diameter & 140 & $\mu \mathrm{m}$ \\
Orifice exit diameter & 112 & $\mu \mathrm{m}$ \\
Orifice fillet radius & 42 & $\mu \mathrm{m}$ \\
Orifice length & 0.988 & $\mathrm{~mm}$ \\
Fuel & Standard & Diesel Elite \\
Injection pressure & 30 & $\mathrm{MPa}$ \\
Ambient pressure & 3.6 & $\mathrm{MPa}$ \\
Ambient temperature & 306 & $\mathrm{~K}$ \\
\hline
\end{tabular}

Table 1: Nozzle geometry and injection conditions.

A mesh sensitivity study was carried out, increasing the internal and external mesh resolutions until differences in mass flow rate and spray tip penetration were lower than $1 \%$. The result is a structured grid of 101424 cells (57420 for the external flow and 44004 inside the nozzle) with 72 elements at the orifice exit.

Injection events are quite short in time, thus phenomena are highly transient in nature as noted by Battistoni et al. (2012). A maximum Courant number (CFL) of 0.6 was used and then the computational time step ranged from $4.010^{-9}$ to $5.010^{-8}$. 
Boundary conditions of the domain, skipping the fuel inlet, are shown in Tables 2-5. Turbulent variables $k$ and $\epsilon$ or $\omega$ boundary conditions depend on the turbulence model and the inlet conditions, and they are not specified in the tables. Nevertheless, velocity fluctuations at the inlet of $5 \%$ are considered and the turbulent length scale is taken as $10 \%$ of the inlet length. $k$ and $\epsilon$ or $\omega$ wall functions are always used to enhance the flow velocity pattern in the logarithmic layer of the boundary layer, and then theoretically obtain velocity at the orifice exit profiles and non-dimensional coefficients closer to the experiments. If no wall function is used, results change less than $1 \%$.

\begin{tabular}{ccc}
\hline Patch & Type & Value \\
\hline outlet & Zero gradient & - \\
wall & Zero gradient & - \\
top & Zero gradient & - \\
\hline
\end{tabular}

Table 2: Boundary conditions for $Y$.

\begin{tabular}{ccc}
\hline Patch & Type & Value \\
\hline outlet & Zero gradient & - \\
wall & Zero gradient & - \\
top & Zero gradient & - \\
\hline
\end{tabular}

Table 4: Boundary conditions for $T$.

\begin{tabular}{ccc}
\hline Patch & Type & Value \\
\hline outlet & Pressure velocity & $\left(\begin{array}{lll}0 & 0 & 0\end{array}\right)$ \\
wall & Fixed value & $\left(\begin{array}{lll}0 & 0 & 0\end{array}\right)$ \\
top & Zero gradient & - \\
\hline
\end{tabular}

Table 3: Boundary conditions for $U$.

\begin{tabular}{ccc}
\hline Patch & Type & Value \\
\hline outlet & Non-reflective & 3634056 \\
wall & Zero gradient & - \\
top & Zero gradient & - \\
\hline
\end{tabular}

Table 5: Boundary conditions for $p$.

In this study, upwind discretization schemes are always used in order to minimize the computational cost (higher order schemes did not significantly increased the accuracy of the solution). An Euler scheme is selected for the time discretization. PBiCG (Preconditioned Bi-Conjugate Gradient) solver with DILU (Diagonal Incomplete LU) preconditioner is used for all variables but the pressure, which is solved by PCG (Preconditioned Conjugate Gradient) solver with DIC (Diagonal Incomplete Cholesky) preconditioner. A relaxation factor of 0.3 is used for the pressure and a factor of 0.7 for the rest of variables but the density, which is solved without relaxation.

Regarding the initialization, all variables are initialized with constant values equal to the outlet ones, except for the liquid mass fraction, which is equal to 1 inside the nozzle and 0 outside. Though it is not completely true, 
it is assumed that the nozzle is filled with liquid because the mixing that takes place inside the nozzle is not modeled.

\subsection{Inlet boundary condition}

When simulating internal flows of diesel injectors, the common choice for the inlet boundary condition is to fix the injection pressure, as Battistoni et al. (2012); Som et al. (2010) and Payri et al. (2013) do. An alternative to avoid pressure wave reflexions that take place in compressible fluids and still being able to fix the pressure is to use a non-reflexive constant pressure boundary condition, often found as outlet boundary condition.

When simulating external flows of diesel injectors, in other words, the spray, the most employed inlet boundary condition is specifying the velocity, which varies with time in order to reproduce the experimental mass flow rate shape, as it is done by Battistoni et al. (2012); Som et al. (2010) and Hoyas et al. (2013). If it is available, the spatial distribution is used, i.e. Battistoni et al. (2012); Som et al. (2010), but if it is not, same velocity value is employed for the whole inlet section, i.e. Hoyas et al. (2013); García-Oliver et al. (2013). The value of the velocity is obtained experimentally by means of the mass flow rate as described by Desantes et al. (2005) and Payri et al. (2012).

Summarizing, three types of inlet boundary condition are tested:

- Constant pressure.

- Non-reflective constant pressure (with different grades of reflexion).

- Time-varying velocity.

Non-reflective pressure condition deserves extra attention. It performs some basic thermodynamic calculations and tries to create a tendency towards the preset value. The larger the grade of reflection, the further the boundary condition will deviate from the specified value, however, the smaller the grade of reflection, the more reflective the boundary tends to be; as OpenFOAM ${ }^{\circledR}$ Corp. (2012) indicates. Thus, 0.01, 0.05 and 0.5 have been tested for values of grade of reflection. 


\section{Results and discussion}

\subsection{Turbulence model}

As stated in Section 1, the five turbulence models are tested with a nonreflective constant pressure inlet boundary condition. Also note before presenting the results that default model constants values are used although other authors have suggested that by modifying some of them the computational results become more accurate, for example Hoyas et al. (2013).

First parameters to be analyzed are the mass flow rate and momentum flux, together with the non-dimensional coefficients that define the nature of the flow of the nozzle $C_{v}, C_{a}$ and $C_{d}$, following Desantes et al. (2005) procedure. Table 6 shows their steady state values compared with experimental ones. SST $k-\omega$ and Realizable $k-\epsilon$ models clearly overestimate all parameters. The reason of that is shown later. RNG $k-\epsilon$ model over-predicts the momentum flux. The other two $k-\epsilon$ models predict well, with a maximum deviation of $3 \%$, the mass flow rate, the momentum flux and non-dimensional parameters.

\begin{tabular}{cccccc}
\hline Turbulence model & $\dot{\boldsymbol{m}}[\mathrm{g} / \mathrm{s}]$ & $\dot{\boldsymbol{M}}[\mathrm{N}]$ & $\boldsymbol{C}_{\boldsymbol{v}}[-]$ & $\boldsymbol{C}_{\boldsymbol{a}}[-]$ & $\boldsymbol{C}_{\boldsymbol{d}}[-]$ \\
\hline Experimental & 1.654 & 0.372 & 0.888 & 0.953 & 0.846 \\
Standard $\boldsymbol{k}-\boldsymbol{\epsilon}$ & 1.700 & 0.370 & 0.869 & 0.942 & 0.819 \\
High density ratio $\boldsymbol{k}-\boldsymbol{\epsilon}$ & 1.701 & 0.375 & 0.874 & 0.943 & 0.824 \\
RNG $\boldsymbol{k}-\boldsymbol{\epsilon}$ & 1.749 & 0.398 & 0.903 & 0.938 & 0.847 \\
Realizable $\boldsymbol{k}-\boldsymbol{\epsilon}$ & 1.992 & 0.500 & 0.998 & 0.967 & 0.965 \\
SST $\boldsymbol{k}-\boldsymbol{\omega}$ & 1.980 & 0.491 & 0.984 & 0.974 & 0.959 \\
\hline
\end{tabular}

Table 6: Steady state and non-dimensional parameters of the turbulence models tested.

Figure 2 shows the velocity profile at the exit of the nozzle. Standard and high density ratio $k-\epsilon$ models have the same profile: small area at the center of the orifice with constant velocity and large area with a parabolic velocity profile, what is characteristic of laminar/low Reynolds number flows. With the RNG $k-\epsilon$ the parabolic region is reduced, and even further reduced with the SST $k-\omega$ or Realizable $k-\epsilon$ models, which show a typical profile of turbulent/high Reynolds number flow as said by Payri et al. (2010, 2012). Because of the current ESA model assumes high Reynolds numbers, these last turbulence model seems better than the others. These velocity profiles explain why $\operatorname{SST} k-\omega$ and Realizable $k-\epsilon$ models give higher mass flow 
rate and momentum flux for the same inlet pressure value. At this point it is worthy to mention that LES models could improve the accuracy on the prediction of the velocity coefficient up to $0.6 \%$ (Payri et al. (2013)). LES models have also been successful in improving primary atomization predictions, Chesnel et al. (2011) evaluated the different available sub-grid models and showed that the scale similarity assumption provides better estimation of the sub-grid terms than the Smagorinsky formulation; however the presence of a constant that needs to be estimated and the various values that are found depending on the filter size are the main drawbacks of this approach.

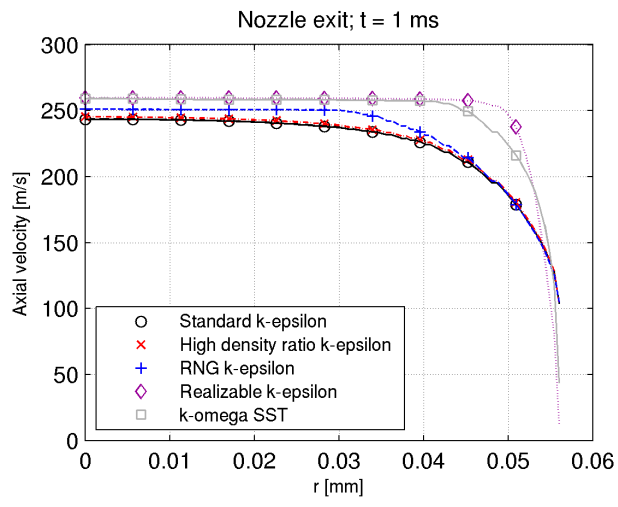

Figure 2: Velocity profile at the exit of the nozzle of the turbulence models tested.

Figure 3 proves that, as expected, standard and high density ratio $k-\epsilon$ models generates the same turbulence levels inside the nozzle. RNG $k-\epsilon$ and SST $k-\omega$ models, which in general are recommended for simulating turbulent wall-bounded flow, produce a completely different turbulent viscosity contour. Furthermore the maximum value is an order of magnitude lower. This maximum value is reached towards the inlet and also next to the wall orifice instead of right at the orifice restriction like with standard and high density ratio $k-\epsilon$ models. This distribution is similar to the one obtained with Large Eddy Simulation (LES) by Payri et al. (2013), a numerical technique which is supposed to be more accurate than RANS. The fifth model, Realizable $k-\epsilon$, generates a turbulent viscosity pattern which is a mixture of the other contours, high turbulence levels in the middle of the orifice but also towards the fuel inlet. Also the maximum value is somewhere in between.

Turbulent viscosity contours of the external flow are plotted in Figure 4. Notice that the scale of the color map is not the same than for the internal 
flow, otherwise some cases would seem to have no gradients. The turbulent intensity generated by standard and high density ratio $k-\epsilon$ models is lower than the generated by the other three models. Since the turbulent viscosity is directly related to the air-fuel mixing (see Equation 2) this figure can also be used to analyze the spray structure. However, Realizable $k-\epsilon$ model does not show the typical spray contour, so it does not seem correct for the external flow of this solver. RNG $k-\epsilon$ model shows a very strange spray structure at the tip which is not seen in the experiments. Standard and high density ratio $k-\epsilon$ seem to correct that behavior but still a kind of "mixing blob" is found at the tip. This "blob" is almost fully eliminated with SST $k-\omega$ model, which gives the typical spray structure experimentally observed.

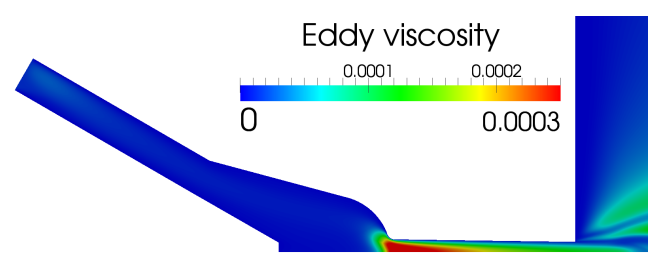

(a) Standard $k-\epsilon$.

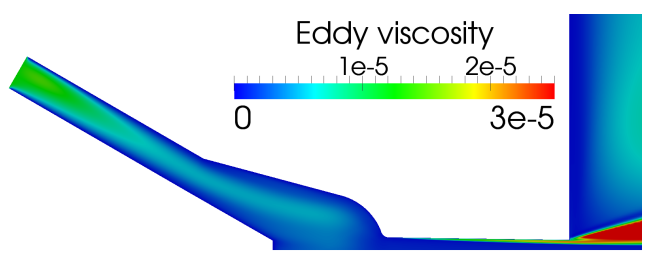

(c) RNG $k-\epsilon$.

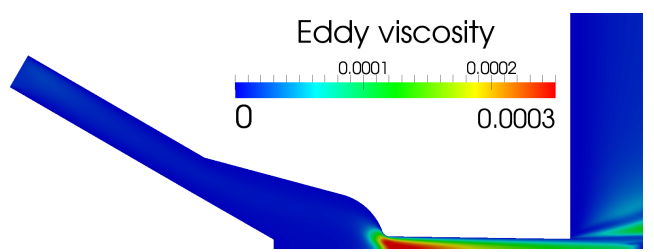

(b) High density ratio $k-\epsilon$.

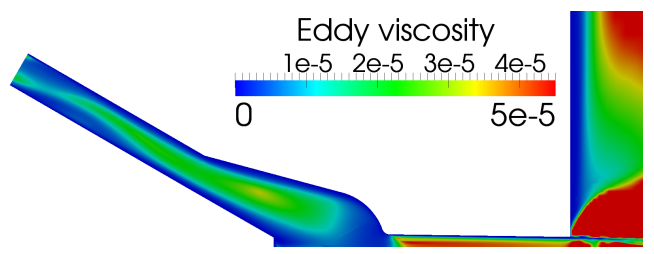

(d) Realizable $k-\epsilon$.

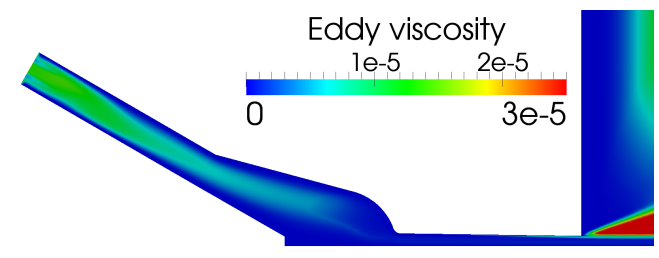

(e) $k-\omega \mathrm{SST}$.

Figure 3: Internal flow turbulent viscosity contours of the turbulence models tested. The time of all images is $1 \mathrm{~ms}$ after start of injection.

Continuing the external flow analysis, Figure 5 shows spray penetration and angle for the five turbulence models tested. It is seen that none of them 


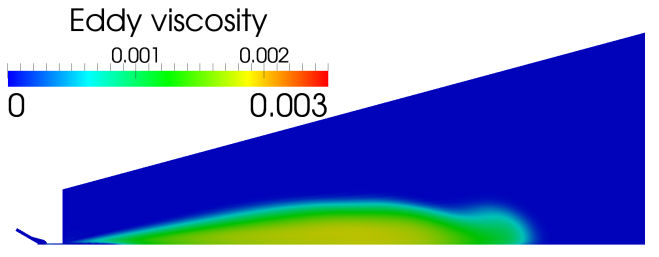

(a) Standard $k-\epsilon$.

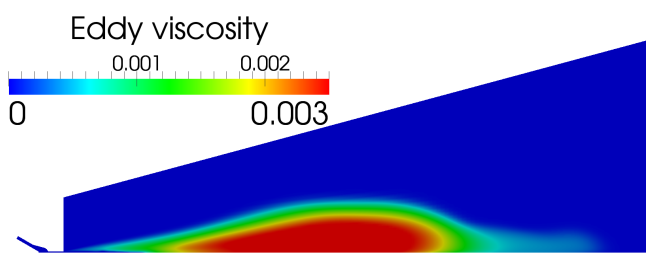

(c) RNG $k-\epsilon$.

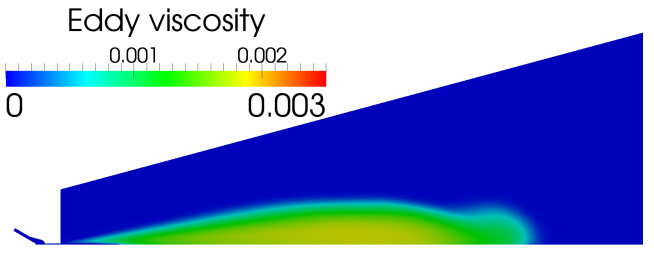

(b) High density ratio $k-\epsilon$.

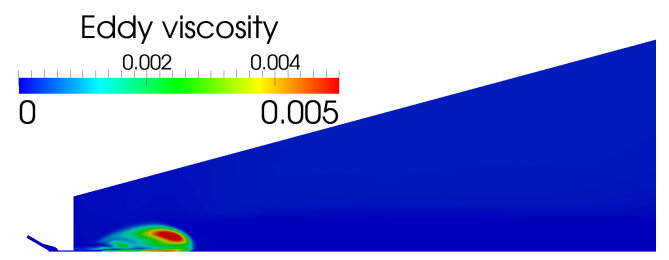

(d) Realizable $k-\epsilon$.

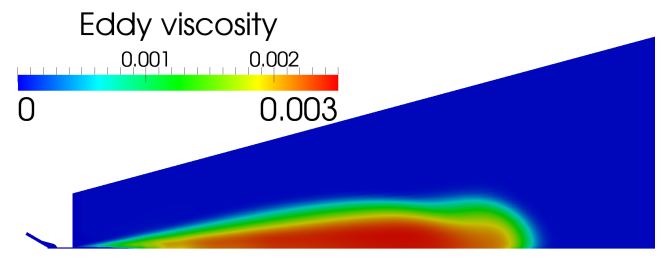

(e) $k-\omega \mathrm{SST}$.

Figure 4: External flow turbulent viscosity contours of the turbulence models tested. The time of all images is $1 \mathrm{~ms}$ after start of injection.

agree with the experimental steady-state spray angle, though this is not really important because the angle strongly depends on the visualization technique which is used, the intensity and quality of the light, the post-processing method of the images (e.g. the threshold), the criteria used for its definition, etc. as noted by Payri et al. (2011b). The spray penetration seems to be well predicted by all models but the RNG and Realizable $k-\epsilon$ models. Probably, adjusting turbulence model constants could fix this discrepancy for the RNG, as done by Hoyas et al. (2013), but not for the Realizable because the spray structure is completely different. Note that in this comparison there is a small temporal delay between experimental and simulated curves due to the fact that the transient initial injection phenomena are not simulated.

Taking into account all the results previously explained, the SST $k-$ $\omega$ turbulence model is the most suitable model among the ones tested for 


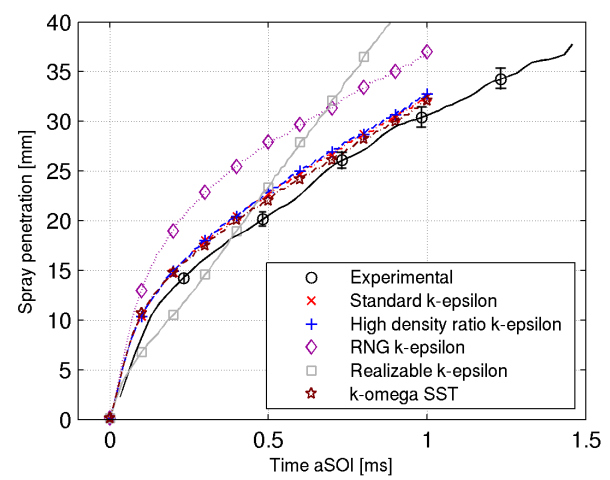

(a) Spray penetration.

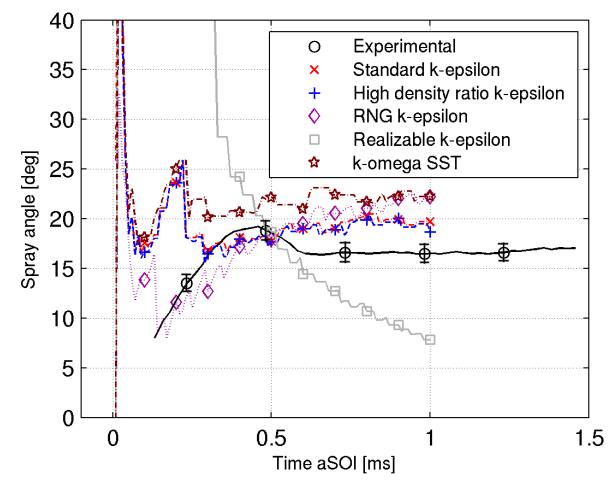

(b) Spray angle.

Figure 5: Spray penetration and spray angle of the turbulence models tested.

the current ESA model. Although it clearly overestimates mass flow rate and momentum flux, the velocity profile at the exit of the orifice fits better with the theoretical one assumed by Payri et al. (2012) and also with the one obtained with LES simulations by Payri et al. (2010). Furthermore, turbulent viscosity contours are more accurate when compared also with LES simulations of Payri et al. (2013). Mismatch in mass flow rate and momentum flux (and their coefficients) when compared to the experiments are probably due to the pressure value at the nozzle inlet which actually has to be lower than in the common-rail due to losses in all conducts and volumes that connect both parts of the injection system, the rail and the injector nozzle. This turbulence model needs to take this into account if pressure inlet boundary condition is going to be used.

\subsection{Inlet boundary condition}

Before presenting the results and just to clarify, all the inlet boundary conditions have been tested with the standard compressible $k-\epsilon$ turbulence model. Table 7 shows mass flow rate, momentum flux and non-dimensional parameters in steady conditions compared with the experimental values. All conditions predict with high accuracy (error lower than 10\%) the corresponding experimental mass flow rate and momentum flux values except for the non-reflective pressure condition with grade of reflexion of 0.50 . This grade of reflexion was rapidly dismissed because it gave values for non-dimensional 
coefficients higher than the unity, which has no physical sense. Regarding the non-dimensional coefficients, all of them are underestimated.

\begin{tabular}{cccccc}
\hline Inlet boundary & $\dot{\boldsymbol{m}}[\mathrm{kg} / \mathrm{s}]$ & $\dot{\boldsymbol{M}}[\mathrm{N}]$ & $\boldsymbol{C}_{\boldsymbol{v}}[-]$ & $\boldsymbol{C}_{\boldsymbol{a}}[-]$ & $\boldsymbol{C}_{\boldsymbol{d}}[-]$ \\
\hline Experimental & 1.654 & 0.372 & 0.888 & 0.953 & 0.846 \\
Constant pressure & 1.638 & 0.347 & 0.843 & 0.941 & 0.793 \\
Mass flow rate & 1.687 & 0.370 & 0.872 & 0.938 & 0.817 \\
Non-reflective 0.01 & 1.648 & 0.352 & 0.849 & 0.941 & 0.799 \\
Non-reflective 0.05 & 1.712 & 0.370 & 0.869 & 0.941 & 0.819 \\
Non-reflective 0.50 & 2.098 & 0.567 & 1.074 & 0.946 & 1.016 \\
\hline
\end{tabular}

Table 7: Steady state and non-dimensional parameters of the inlet boundary conditions tested.

Not only steady state values are important, also the time evolution of mass flow rate is of interest. It is plotted in Figure 6. It is clearly observed how non-reflective pressure condition with grade of reflexion of 0.50 gives a growing mass flow rate, coming from a continuous growing of the inlet pressure. Thus, its values given in Table 7 are not steady state and cannot be compared to the experimental ones. This result also confirms that large values of grade of reflection are not correct and must be dismissed, which is an interesting result because this type of boundary condition is selected for the outlet.

When comparing constant pressure conditions (including non-reflective) with mass flow rate condition the expected result is observed: with pressure conditions the steady state is reached much faster meanwhile the mass flow rate of time-varying velocity condition follows exactly the imposed trend, which is the experimental one.

Differences between constant pressure and non-reflective pressure can be seen also in Figure 6. Two observations can be drawn. First one concerns the oscillations at the inlet for the constant pressure condition, which are damped within the nozzle and disappear with time. They are generated by the pressure wave that rebounds and travels downwards to the orifice entry (a restriction) and upwards to the inlet. Regardless the physical sense of these oscillations, such temporal gradients could lead to numerical divergence. Obviously, they are not present with non-reflective boundary condition. The second observation corresponds to a difference in mass flow rate. Non-reflective pressure condition gives a slightly higher value than constant 


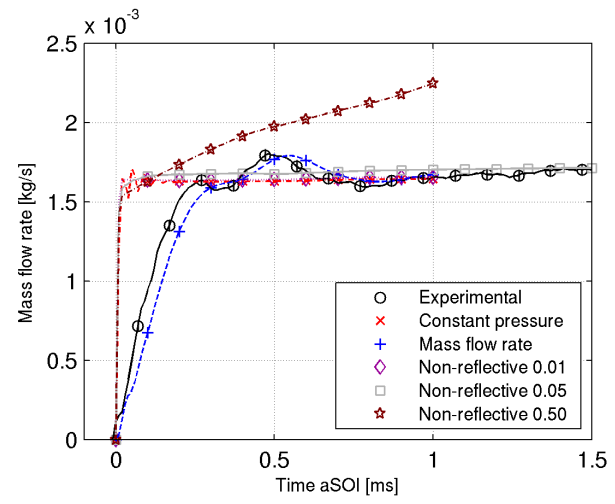

Figure 6: Mass flow rate of the inlet boundary conditions tested.

pressure, and this is due to the deviation in the pressure from the imposed value.

The shift between experimental and mass flow rate curves in Figure 6 is also worthy to mention. Although the mass flow rate is imposed at the inlet, both curves do not superpose. This is due to compressibility effects that take place inside the nozzle. As the liquid is a compressible fluid, pressure waves travel up and down through the nozzle accelerating the fluid, and it takes some time until the right velocity in every section of the nozzle is reached. In future studies, these effects can be taken into account before starting the simulation and set a mass flow rate at the nozzle inlet that gives exactly the experimental curve at the orifice exit.

So far, only the internal flow has been analyzed. However a change on the inlet boundary condition also affects external flow parameters such spray penetration or spray angle, plotted in Figure 7. All constant pressure conditions (included non-reflective ones) present the same penetration and spray angle; and the mass flow rate boundary condition gives also similar values but shifted in time. A very interesting result is the behavior of the penetration very short time after the start of injection given by the mass flow rate condition. This change in the slope is not present in the experimental curve because no measurements in the near field were made by Payri et al. (2011b), notwithstanding other authors have experimentally observed it, for example, de la Morena (2011). Despite that, the behavior is similar to the experimental curve, maybe with slightly higher slope.

Spray angle steady state is again not in agreement with the experimental 


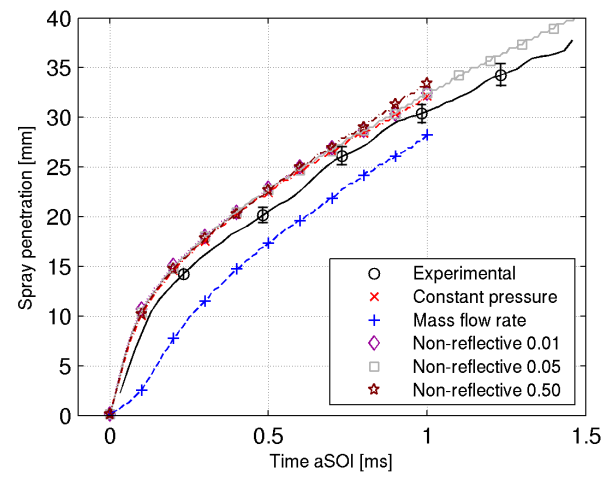

(a) Spray penetration.

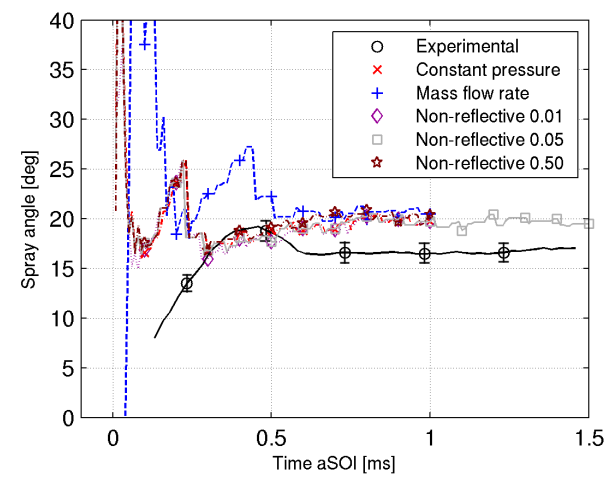

(b) Spray angle.

Figure 7: Spray penetration and spray angle of the inlet boundary conditions tested.

value, but the experimental behavior is captured by all inlet boundary conditions, though constant pressure conditions (included non-reflective) with a small temporal lead for the peak.

Taking into account all the results previously explained, the mass flow rate boundary condition (time-varying velocity) is the most suitable boundary condition for the inlet among the tested ones for the current ESA model. This condition estimates well the momentum flux and spray tip penetration without oscillations which could lead to divergence. The main drawback of this boundary condition is that the mass flow rate has to be previously experimentally measured.

\subsection{Combination of turbulence model and inlet boundary condition}

After analyzing the turbulence model and the inlet boundary condition separately, it is necessary to check that the combination of both accurately represents the experimental results.

First, steady state mass flow rate, momentum flux and non-dimensional coefficients obtained with SST $k-\omega$ turbulence model and time-varying velocity inlet boundary condition are compared with the experimental values in Table 8. It is seen that: the momentum flux and the velocity coefficient $C_{v}$ are about a $4 \%$ lower than the experimental values, the error in the area coefficient $C_{a}$ is lower than $2 \%$, and the predicted discharge pressure $C_{d}$ is about $3 \%$ smaller than the experimental one. This good agreement is kind of expected because the mass flow rate is imposed. 


\begin{tabular}{cccccc}
\hline Case & $\dot{\boldsymbol{m}}[\mathrm{g} / \mathrm{s}]$ & $\dot{\boldsymbol{M}}[\mathrm{N}]$ & $\boldsymbol{C}_{\boldsymbol{v}}[-]$ & $\boldsymbol{C}_{\boldsymbol{a}}[-]$ & $\boldsymbol{C}_{\boldsymbol{d}}[-]$ \\
\hline Experimental & 1.654 & 0.372 & 0.888 & 0.953 & 0.846 \\
Computational & 1.692 & 0.361 & 0.847 & 0.968 & 0.820 \\
\hline
\end{tabular}

Table 8: Steady state and non-dimensional parameters with SST $k-\omega$ turbulence model and time-varying velocity inlet boundary condition.

Second, spray tip penetration and spray angle obtained with the simulation can be compared with the experimental curves, as done in Figure 8. The experimental penetration curve has been moved in time $50 \mu \mathrm{m}$ to match its start with the computational curve. This can be done because, as commented before, although the experimental mass flow rate is imposed the transient phenomena during start of injection is not modeled. Furthermore, initial conditions of the internal flow are not hundred per cent right, in reality the velocity is not zero when the whole nozzle is filled with liquid. It is seen that the spray penetrates faster than it is predicted by the model. Regardless this temporal mismatch, curve slopes after $0.4 \mathrm{~ms}$ of simulation are exactly the same and also values are the same but shifted in time. The spray angle is again overestimated by the model. Nevertheless, the trend is well captured.

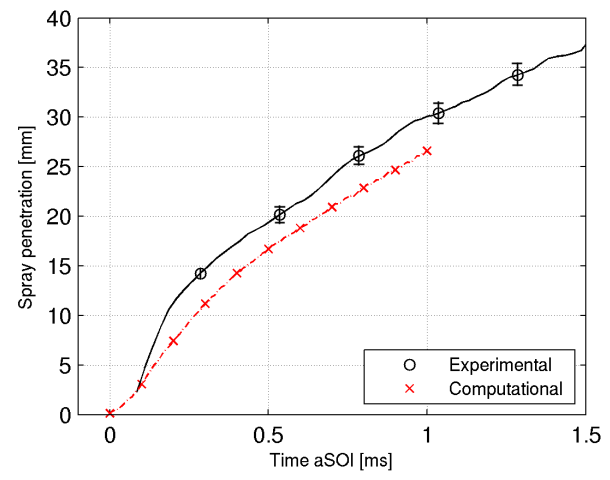

(a) Spray penetration.

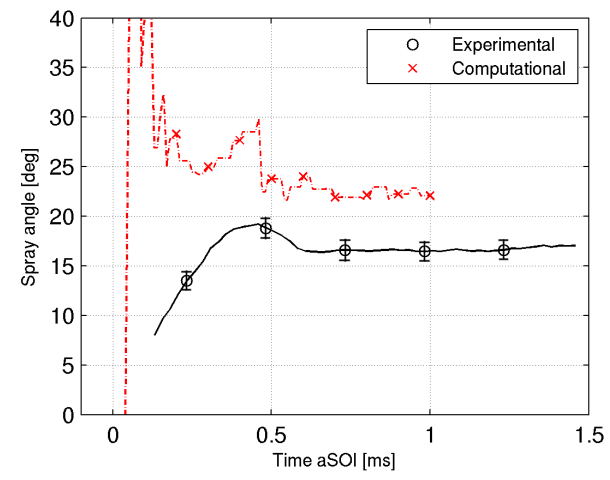

(b) Spray angle.

Figure 8: Spray penetration and spray angle with SST $k-\omega$ turbulence model and timevarying velocity inlet boundary condition.

And third, experimental images are compared with mass fraction con- 
tours in order to check if the spray structure is well reproduced. Figure 9 shows four different time steps. In each one, the upper part corresponds to the liquid mass fraction contour and the lower part to the experimental spray contour obtained via back-light illumination technique by Payri et al. (2011b). The time of experimental images is about 150-180 $\mu$ s delayed in order to compare images with the same penetration value. A very good general agreement is found. The tip "mixing blob" is present in both cases and the chosen turbulence model predicts its size pretty well. The only aspect of the spray that is not well captured is the angle, as it has been previously shown. Especially near the nozzle, the spray obtained with the ESA model is wider than the one experimentally observed. However, this part of the spray is tricky and a better comparison with near nozzle experiments is required to draw further conclusions.

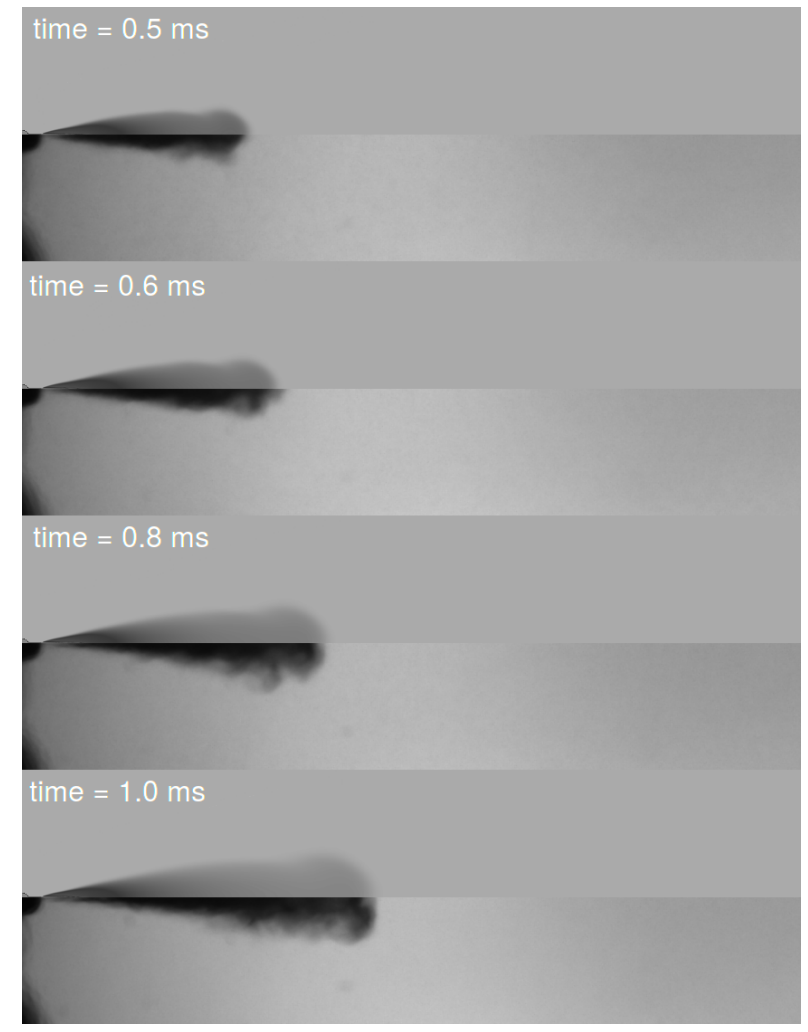

Figure 9: Spray time evolution. Comparison with experimental results of Payri et al. (2011b). The timing corresponds to the simulation. 


\section{Conclusions}

The $\Sigma-Y$ model for simulating break-up and liquid-air mixing processes developed by Vallet et al. has been modified in order to make it work for both internal and external flows in the field of diesel injection. The modification has been called Eulerian Spray Atomization (ESA) model.

Several turbulence models among the most common types have been tested for a single-hole nozzle. Surprisingly, a model generally recommended for wall-bounded flow such SST $k-\omega$ has been found to give accurate results not only on the internal flow but also on the spray development.

Separately, several inlet boundary conditions have been also tested. It turns out that the common solution taken for the external flow of fixing a time-varying velocity also works out when the internal domain is added.

The combination of above findings lead to a solution with an error lower than $5 \%$ when compared to experimental values for all internal flow parameters and spray tip penetration. However, the value of the spray angle is not well predicted and the computational spray is slightly wider.

\section{Acknowledgements}

This research was funded in the frame of project "Compresión de la influencia de combustibles no convencionales en el proceso de inyección y combustión tipo Diésel" reference TRA2012-36932 from Ministerio de Economía y Competitividad (Spanish Ministry of Economy).

\section{References}

Arienti, M., Sussman, M., 2014. An embedded level set method for sharpinterface multiphase simulations of Diesel injectors. International Journal of Multiphase Flow 59, 1-14. doi:10.1016/j.ijmultiphaseow.2013.10.005.

Bardi, M., Payri, R., Malbec, L.M., Bruneaus, G., Picket, L.M., Manin, J., Bazyn, T., Genzale, C.L., 2012. Engine Combustion Network (ECN): Comparison of Spray Development, Vaporization and Combustion in Different Combustion Vessels. Atomization and Sprays 22, 807-842. doi:10.1615/AtomizSpr.2013005837.

Battistoni, M., Grimaldi, C., Mariani, F., 2012. Coupled Simulation of Nozzle Flow and Spray Formation Using Diesel and Biodiesel for CI Engine Applications. SAE International 2012-01-1267 doi:10.4271/2012-01-1267. 
Befrui, B., Corbinelli, G., Spiekermann, P., Shost, M., 2012. Large Eddy Simulation of GDI Single-Hole Flow and Near-Field Spray. SAE International 2012-01-0392 doi:10.4271/2012-01-0392.

Beheshti, N., Burluka, A.A., Fairweather, M., 2007. Assesment of $\Sigma-Y_{\text {liq }}$ model predictions for air-assisted atomization. Theoretical and Computational Fluid Dynamics 21, 381-297. doi:10.1007/s00162-007-0052-3.

Belhadef, A., Vallet, A., Amielh, M., Anselmet, F., 2012. Pressure-swirl atomization: modeling and experimental results. International Journal of Multiphase Flow 39, 13-20. doi:10.1016/j.ijmultiphaseflow.2011.09.009.

Bermúdez, V., Payri, R., Salvador, F.J., Plazas, A., 2005. Study of the influence of nozzle seat type on injection rate and spray behavior. Proceedings of the Institution of Mechanical Engineers, Part D, Journal of Automobile Engineering 219, 677-689. doi:10.1243/095440705X28303.

Blokkeel, G., Barbeau, B., Borghi, R., 2003. A 3D Eulerian model to improve the primary breakup of atomizing jet. SAE Technical Paper 2003-01-005 doi:10.4271/2003-01-0005.

Chesnel, J., Ménard, T., Réveillon, J., Demoulin, F.X., 2011. Subgrid Analysis of Liquid Jet Atomizatoin. Atomization and Sprays 21, 41-67.

Corp., S.G.I., 2012. OpenFOAM. User Guide. http://www.opencfd.co.uk. Available online.

Demoulin, F.X., Beau, P.A., Blokkeel, G., Mura, A., Borghi, R., 2007. A new model for turbulent flows with large density fluctuations: application to liquid atomization. Atomization and Sprays 17, 315-345. doi:10.1615/AtomizSpr.v17.i4.20.

Desantes, J.M., Payri, R., Pastor, J.M., Gimeno, J., 2005. Experimental characterization of internal nozzle flow and diesel spray behavior. Part 1: Non-evaporative conditions. Atomization and Sprays 15, 489-516.

Duret, B., Reveillon, J., Menard, T., Demoulin, F.X., 2013. Improving primary atomization modeling through DNS of two-phase flows. International Journal of Multiphase Flow 55, 130-137. doi:10.1016/j.ijmultiphaseflow.2013.05.004. 
García-Oliver, J.M., Pastor, J.M., Pandal, A., Trask, N., Baldwin, E., Schmidt, D.P., 2013. Diesel spray CFD simulation based on the $\Sigma-$ $Y$ eulerian atomization model. Atomization and Sprays 23, 71-95. doi:10.1615/AtomizSpr.2013007198.

Hoyas, S., Gil, A., Margot, X., Khuong-Anh, D., Ravet, F., 2013. Evaluation of the Eulerian-Lagrangian Spray Atomization (ELSA) model in spray simulations: 2D cases. Mathematical and Computer Modelling 57, 16861693. doi:10.1016/j.mcm.2011.11.006.

Lebas, R., Blokkeel, G., Beau, G., Demoulin, F.X., 2005. Coupling vaporization model with the Eulerian-Lagrangian Spray Atomization (ELSA) model in diesel engine conditions. SAE Technical Paper 2005-01-0213 doi:10.4271/2005-01-0213.

Lebas, R., Menard, T., Beau, P., Berlemont, A., Demoulin, F.X., 2009. Numerical simulation of primary break-up and atomization: DNS and modeling study. International Journal of Multiphase Flow 35, 247-260. doi:10.1016/j.ijmultiphaseflow.2008.11.005.

Lee, C.H., Reitz, R.D., 2013. CFD simulations of diesel spray tip penetration with multiple injections and with engine compression ratios up to 100:1. Fuel 111, 289-297. doi:10.1016/j.fuel.2013.04.058.

Marcer, R., Cottier, P.L., Chaves, H., Argueyrolles, B., Habchi, C., Barbeau, B., 2000. A Validated Numerical Simulation of Diesel Injector Flow Using a VOF Method. SAE Technical Paper 2000-01-2932 doi:10.4271/2000-012932.

de la Morena, J., 2011. Estudio de la influencia de las caractersticas del flujo interno en toberas sobre el proceso de inyección diésel en campo próximo. Ph.D. thesis. Departamento de Máquinas y Motores Térmicos, Universitat Politècnica de València.

Ning, W., Reitz, R.D., Diwakar, R., Lippert, A., 2009. An Eulerian-Lagrangian spray and atomization model with improved turbulence modeling. Atomization and Sprays 19, 727-739. doi:10.1615/AtomizSpr.v19.i8.20.

Payri, R., Gimeno, J., Martí-Aldaraví, P., Bracho, G., 2013. Study of the influence of the inlet boundary conditions in a les simulation of internal 
flow in a diesel injector. Mathematical and Computer Modelling 57, 17091715. doi:10.1016/j.mcm.2011.11.019.

Payri, R., Salvador, F.J., Gimeno, J., Bracho, G., 2011a. The effect of temperature and pressure on thermodynamic properties of diesel and biodiesel fuels. Fuel 90, 1172-1180. doi:10.1016/j.fuel.2010.11.015.

Payri, R., Salvador, F.J., Gimeno, J., García, A., 2012. Flow regime effects over non-cavitating injection nozzles. Proceedings of the Institution of Mechanical Engineers, Part D, Journal of Automobile Engineering 226, 133-144. doi:10.1177/0954407011413056.

Payri, R., Salvador, F.J., Gimeno, J., Novella, R., 2011b. Flow regime effects on non-cavitating injection nozzles over spray behavior. International Journal of Heat and Fluid Flow 32, 273-284. doi:10.1016/j.ijheatfluidflow.2010.10.001.

Payri, R., Salvador, F.J., Gimeno, J., Soare, V., 2005. Determination of diesel spray characteristics in real in-cylinder air density and pressure conditions. Journal of Mechanical Science and Technology 19, 2040-2052. doi:10.1007/BF02916497.

Payri, R., Tormos, B., Gimeno, J., Bracho, G., 2010. The potential of Large Eddy Simulation (LES) code for the modeling of flow in diesel injectors. Mathematical and Computer Modelling 52, 1151-1160. doi:10.1016/j.mcm.2010.02.033.

Salvador, F.J., Martínez-López, J., Caballer, M., de Alfonso, C., 2013. Study of the influence of the needle lift on the internal flow and cavitation phenomenon in diesel nozzle by CFD using RANS methods. Energy Conversion and Management 66, 246-256. doi:10.1016/j.enconman.2012.10.011.

Som, S., Longman, D.E., Ramírez, A.I., Aggarwal, S.K., 2010. A comparison of injector flow and spray characteristics of biodiesel with petrodiesel. Fuel 89, 4014-4024. doi:10.1016/j.fuel.2010.05.004.

Vallet, A., Burluka, A.A., Borghi, R., 2001. Development of a Eulerian model for the "atomization" of a liquid jet. Atomization and Sprays , 619-642. 
Weller, H.G., Tabor, G., Jasak, H., Fureby, C., 1998. A tensorial approach to computational continuum mechanics using object-oriented techniques. Computer in Physics 12, 620-631. doi:10.1063/1.168744. 